\title{
The use of modal parameters in structural health monitoring
}

\author{
Ali Al-Ghalib ${ }^{1, *}$, and Fouad Mohammad ${ }^{2}$ \\ ${ }^{1}$ College of Engineering, Al-Mustansiriyah University, Baghdad, Iraq \\ ${ }^{2}$ School of Architecture, Design and the Built Environment, Nottingham Trent University, Nottingham, UK
}

\begin{abstract}
The concrete is liable to damage due to various stresses which compensate its adequacy and safety. The estimation of remaining strength in reinforced concrete beams when subjected to increased loading action utilizing vibration parameters is investigated. For this reason, three beams are loaded statically close to failure in various increasing load steps and then repaired. The beams are all of same dimensions, but are different in strength and range of defects introduced to each sample. Following each loading step, the experimental modal testing is utilized to collect the vibration parameters (natural frequency, damping ratio and mode shapes) of each beam when tested under free support boundary conditions. The use of vibration parameters for the purpose of damage identification are known to be an elaborate and lengthy process. On the other hand, they are successful for the structural health monitoring given that they are able to provide global on-site automated continuous monitoring. The paper features post analysis procedures for experimental modal measurements of three concrete samples to obtain and correlate the basic modal parameters (natural frequency, modal damping and mode shapes). The results of the extracted modal parameters and their combination are exploited in this research as quantified identification parameters. This paper concludes that modal parameters are successful in determining the location and quantity of structural degradation, when holistic approach considered through a system.
\end{abstract}

\section{Introduction}

Thenumber of aged and damaged civil engineering infrastructure has increased significantly in the past few decades, and therefore, the subject of structural health monitoring (SHM) becomes the focus of many researchers. Much research concentrates on applying an automated monitoring method that replaces or complements the current onerous visual inspection and other local non-destructive testing (NDT) methods. Important part of research has particularly given to study the vibration-based paradigms for damage identification purposes [1 and 2].

The vibration-based monitoring systems are developed and constantly adapted to the monitoring of civil engineering structures. These systems are advantageous of global and automated nature. Quantitative analysis on any type of structure can be performed by using the assembled measurements from these systems. It is widely accepted that these are the only methods can present a mathematical model for the actual structure [3]. The possible combination of the theoretical model together with the experimental measurements makes these vibration-based methods more enticing. The current wide interest in the use of monitoring systems in the SHM has been attributed to ${ }^{1}$ the advancement and low expenses of measurement facilities [4].
The concept of arranging the damage identification assessment in four levels, namely: detection, localization, quantification and prediction was first proposed by Rytter in his doctoral study back in 1993 [4 and 5].

The identification of damage in RC beams inflicted with progressive cracking behavior by utilizing vibration data is considered difficult and is still unpredictable in the published literature. Therefore, this research endeavors to explore the extent of some vibration parameters for damage identification when applied to reinforced concrete (RC) beams. For this purpose, three RC beams of laboratory size were cast and set to various damage situations. For each particular state condition the Experimental Modal Analysis (EMA) was implemented for the beam under free-free support condition. An appropriate post analysis procedure for EMA measurement of the three RC beams was conducted in order to obtain the natural frequencies, damping ratios and mode shapes. The study exploits the modal parameters and their combinations in order to interpret changes in vibration properties with the corresponding drop in bending stiffness. The facts established in this research can be widened to involve large scale structures. Having experienced the advantage of the vibration-based techniques in SHM, the author wishes to share the concepts of these prominent systems with the engineering community in Arab and Middle East regions.

\footnotetext{
* Corresponding author: ali.alghalib@,uomustansiriyah.edu.iq
} 


\section{Laboratory tests}

\subsection{Characteristics of concrete beams}

The degree of damage was captured and evaluated through testing three RC beams modeled with the following levels of faults.

All the three test beam samples were of the same dimensions, which are $2.0 \mathrm{~m}$ long, $140 \mathrm{~mm}$ deepand $90 \mathrm{~mm}$ wide. The first beam sample (Beam A) has been investigated under seven loading cases: intact, $3 \mathrm{kN}$ load, $6 \mathrm{kN}, 10 \mathrm{kN}, 13 \mathrm{kN}, 16 \mathrm{kN}$ and externally repaired. Second sample (Beam B) has been investigated under same loading conditions as for Beam A. However, the third sample (Beam C) has been investigated under three different statuses, that is: intact, $10 \mathrm{kN}$ and $14.5 \mathrm{kN}$ load.

The load status and hence, the related cracking conditions have beencreated by using the procedure of four-point static load. Table 1 shows the dimensions and physical properties of the three beams. The experimental investigations of the beams as well as the dynamic test results represent some outcomes of the author's PhD thesis [6].

\subsection{Vibration test}

One of the main issues could be faced in vibration tests is the interaction of support conditions and its influence on the measurements. Therefore, in small scale samples, a freely supported testing model is usually chosen so as to remove the effect of support rigidity [7]. According to this point, the RC beams have been tested in a free-free support condition, as shown in Fig. 1. In this procedure, each beam has been hung by the means of elastic cords attached at $0.46 \mathrm{~m}$ from each end. Three accelerometers have been fixed beginning from the one end at stations $0.2 \mathrm{~m}$ apart. These three accelerometers then moved to fresh three points to cover the whole length with a total of 11 measurement points. The beam has been hit and translation accelerations considered normal to the direction of the elastic cords. The Brüel\&Kjaerimpact hammer type 8208 generated a dynamic force with a sensitivity of $0.0002 \mathrm{~V} / \mathrm{N}$ containing frequency components up to $1.0 \mathrm{kHz}$. The first four modes been covered with the force excitation. The response measurements collected using Brüel\&Kjaer 4514 dynamic acceleration sensor with sensitivity of $0.00985 \mathrm{~V} / \mathrm{g}$ and a frequency range up to $10000 \mathrm{~Hz}$. Both output (response) and input (force) signals have been captured using a data acquisition system of 4100 samples, taken at a rate of 8180 samples per second.

\subsection{Experimental modal analysis}

The EMA is a dynamic test whereby it is generally utilised to obtain the specifications of the structural elements with regard to the modal parameters. In this particular study and for each measurement point, the amplification, filtration and transformation into frequency domain have been conducted to independent four force and response time signals.

The accuracy of Frequency Response Function (FRF) from four averages was checked through constant checking of features, including repeatability and reciprocity.

Table 1. Specifications of The Three Test RC Beams

\begin{tabular}{|l|l|l|l|l|}
\hline $\begin{array}{l}\text { Test } \\
\text { Beam }\end{array}$ & $\begin{array}{l}\text { Cube } \\
\text { strength } \\
\mathbf{N} / \mathbf{m m}^{2}\end{array}$ & $\begin{array}{l}\text { Mass } \\
\mathbf{k g} / \mathbf{m}^{3}\end{array}$ & $\begin{array}{l}\mathbf{E}_{\mathbf{d}}{ }^{*} \mathbf{1 0}^{\mathbf{3}} \\
\mathbf{M P a}\end{array}$ & $\mathbf{G}_{\mathbf{d}}{ }^{*} \mathbf{1 0}^{\mathbf{3}} \mathbf{M P a}^{\dagger}$ \\
\hline Beam A & 20.4 & 2240 & 19.1 & 8.13 \\
\hline Beam B & 29.0 & 2270 & 27.5 & 11.5 \\
\hline Beam C & 27.3 & 2320 & 25.0 & 10.4 \\
\hline
\end{tabular}

${ }^{*} \mathrm{E}_{\mathrm{d}}$ : Dynamic (tangent) modulus of elasticity.

${ }^{\dagger} \mathrm{G}_{\mathrm{d}}$ : Dynamic (tangent) shear modulus of elasticity.

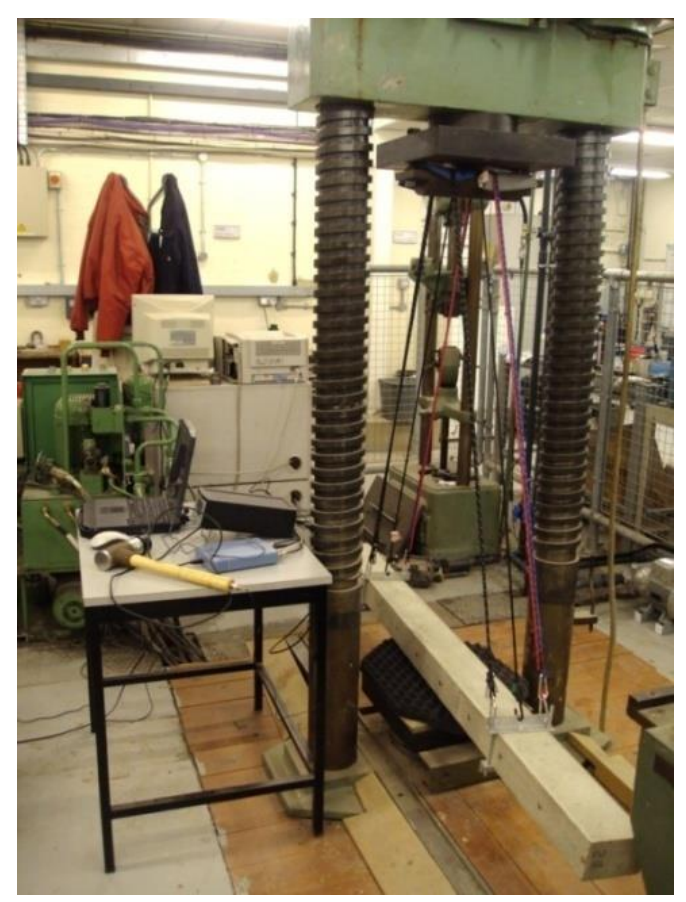

Fig. 1. Freely supported RC beam under dynamic test.

The coherence functions, which measure the correlation between the output and the input signals [8], and are used as a dataverification procedure, were exploited in this study for the FRFs of the system.

In the post-test analysis stage, FRF measurements are basically analysed so as to find the modal parameters of the structures. The simplest modal post-test analysis method is known as peak-picking method, and it is used along with the single degree of freedom systems to obtain the modal parameters. In this method, the response measurements are apparently controlled by resonant modes at their corresponding natural frequency [9], which is the case in this study. 


\section{Damage identification using modal parameters}

\subsection{Effect of damage on natural frequency}

The frequency corresponding to the peak amplitude of the FRF is taken as the resonance frequency $(\omega)$ of a particular vibration mode. For each one of the three beam samples and for all damage conditions, the results of the natural frequencies for the first bending modes were found. The progressive of cracks in concrete beams will cause a drop in the stiffness, and hence, the drop stiffness is manifested as a decrease in the natural frequencies.

For Beams A, B, and C, the outcomes of frequency variation of every loading status from the reference frequency of the intact status are shown in Fig. 2. The variation in frequencies for the first mode is noteworthy compared to the variation in higher modes. This observation suggests that the first mode of vibration is influenced more by the crack pattern compared to the higher modes.

For instance in Beam A, close to the complete failure stage $(\mathrm{P}=16 \mathrm{kN})$, the first four bending frequencies dropped respectively by $20.6 \%, 11.8 \%, 9.6 \%$ and $11.6 \%$ from the intact frequency. The first bending frequency corresponding to $6 \mathrm{kN}$ load case drops about $6 \%$ from the intact first frequency, even though the beam shows no signs of visual cracks. The first bending frequency resumed $7.2 \%$ of frequency variation when retrofitting work was done to the beam.

The modal frequencies of sample $\mathrm{B}$ were considerably dropped with the development of cracks of the increased loading compared with beforehand beam sample (beam A), as shown in Fig. 2. The number and width of cracks driven through the loading steps were ample. This behaviour was manifested as bigger drops in frequencies.

When the beam was loaded with $6 \mathrm{kN}$, the first four frequencies decreased by $8.8 \%, 3.8 \%, 4.6 \%$, and $3.3 \%$, respectively compared to the initial intact state. The big differences in natural frequencies conclude that vibration-based methods and natural frequencies (in particular) are able to detect faults at its early stage, without any apparent visible change. In additions, the first four natural frequencies of the four bending modes decreased respectively by $30.5 \%, 18.9 \%, 14.6 \%$ and 16.5 , when the beam was loaded by $(\mathrm{P}=15.5 \mathrm{kN})$.

For Beam C, the discrepancy of frequencies with growth of cracks is shown in Fig. 2. The measured frequencies of the first four modes decreased continuously responding to the increasing damage.

The abovementioned results can be generalized to draw threshold by which the structure is considered risky when the variation in frequencies of first modes drop to a certain level. It is fortunate that results similar to those of present study were reported in other research employed to real size RC structures [10,11, and 12].
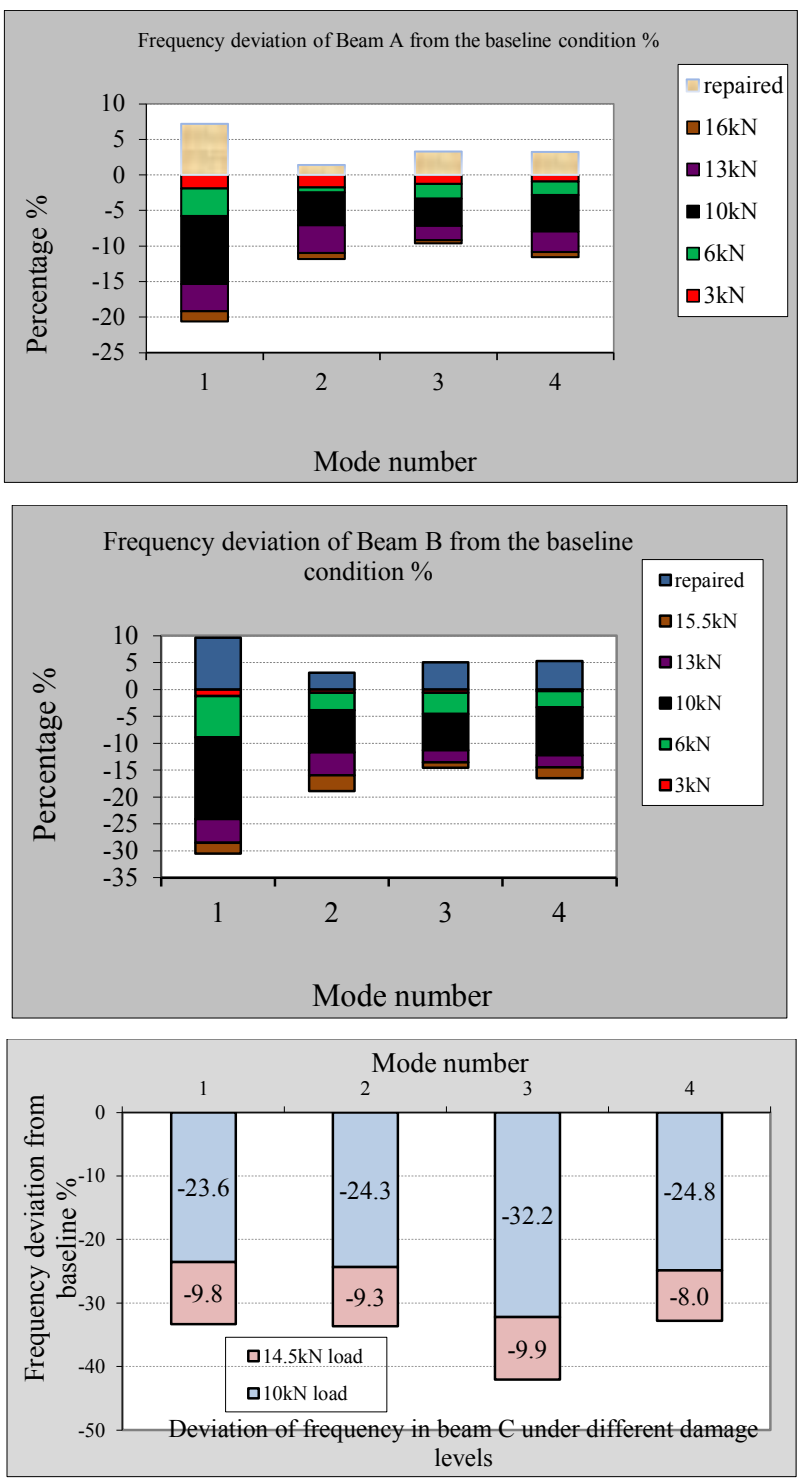

Fig. 2. Variation of natural frequencies for beams A, B and C for different damage conditions.

\subsection{Effect of damage on modal damping ratio}

Of the various modal parameters, damping ratio is supposedly the less reliable quantity to estimate because it is quite sensitive to the background noise. The modal damping ratios of the current study were estimated from the width of the resonance peak, because the beams are lightly damped elements. The half-power bandwidth technique is utilized herein because it is common, quick and widely practiced [13].

The usual presence of cracks in structural elements often increases the amount of the energy dissipation. This phenomenon will affect and control the height and width of peaks of the FRF measurements.

After a round of experiments and calculations, the normalised modal damping ratios for Beam $\mathrm{A}$ for the four modes are given in Fig. 3. Although the evaluation of damping ratios is error-prone, consistent results for modal damping were found. For the consecutive loading steps, changes in modal damping ratios were regular as 
they increased with the magnitude of load. The modal damping ratios gone higher by $39 \%, 6.3 \%, 33 \%$, and $78.1 \%$ for the four modes of vibration, respectively compared to the intact beam when the beam was loaded to $16 \mathrm{kN}$ load. The non-regular rounds of application of impact forces in magnitude, direction and position could as well cause unexpected behavior in damping results. It might seem illogical for the rate of change of damping with the gradual development of cracks appeared nonuniform because the damping ratios were estimated assuming linear structure fitted to non-linear vibration frequency functions.

The Beam B showed clearer changes than the case of Beam A. This beam exhibited obvious increase in modal damping ratios with the developed damage. In Fig. 3, the normalised damping ratios for the first four bending modes with evolution of damage are given in reference to the intact beam. At the final loading stage whereby $\mathrm{P}=15.5 \mathrm{kN}$, the modal damping increased in the order of $143 \%, 96 \%, 114 \%$, and $141 \%$ for the four modes compared to the virgin beam stage. Similar behaviour to that described in section $\mathrm{A}$, trends of modal damping for the first mode increased significantly responding to the increasing damage.

Obviously, for the retrofitted beam, the first four damping ratios decreased by $40 \%, 33 \%, 23 \%$ and $31 \%$ from the severely damaged $(15.5 \mathrm{kN})$ load stage for the four modes, respectively. The effect of modification added to the repaired beam was sufficiently exposed.

When a four-point static load of $10 \mathrm{kN}$ was carried out to beam $\mathrm{C}$, intrinsic distributed cracks was experienced, and this increased the damping ratios significantly, as shown in Fig. 3. For this load step, the modal damping ratios were increased by $60 \%, 8 \%, 213 \%$ and $555 \%$ from the virgin beam reference line for the four modes, respectively.

\subsection{Effect of damage on mode shape}

The third outcome in the modal analysis campaign that is the mode shapes of vibration was further assessed as damage identification factor. Prior to the identification process, the main mode shapes have to be taken out from FRF measurements. One of the approaches used for extracting the mode shapes, where a little effort is needed, is the approach known as the (Quadrature Picking). In this approach a light pairing between the modes is assumed [14]. The observed mode shapes of the three beams and for all loading steps were generated all through in this work.

In view of state deterioration of a structure, the change in the amount and/or shape of a mode shape reflects change in strength. The evaluation of various mode shapes represent different state conditions with respect to one of virgin model, qualitative information is likely to achieve regarding the site and scale of structural changes [13]. Meanwhile, the effect of the environmental conditions has little effect on the mode shapes compared to their effect on frequencies [13].
As anexample to exercise the ability of mode shapes to localize the damage, Fig. 4 shows the overlaid first four flexural mode shapes for beam A.The damage was very noticeable in the first and second mode shapesin comparison to the third and fourth mode shapes.

As well, different damage statuses were sufficiently recognized by those mode shapes. The mode shape for the retrofitted beam managed to place the associated status within its reasonable place. Even though damage (change in condition) was light, the change of mode shape was light as well but subtle.

The changes are liable to become larger with damage level. However, the mode shapes of the first bending mode shape for the beam A produced the most substantial changes in the magnitude of mid span where visual inspection gave no indication of cracking at early loading stages. In addition, the changes of mode shape were not restricted at the damaged area of the beams, but were distributed over the entire length. Results of mode shapes from investigations on pre-stressed concrete bridge girder performed by Huth et al. [15] and results of this study are alike.

\section{Damage quantification via stiffness calculation}

\subsection{Theory}

The work of Maeck and De Roeck[16] was concluded that the location and size of damage in one-dimensional element could be estimated by using the curvature of mode shape instead of the deflection curve. The method, which is named: Direct Stiffness Calculation (DSC), exploits two modal parameters to identify the distributed rigidity in an element, which are natural frequency and mode shape. The basic bending-curvature relationship of the beam element is used for this objective. Certainly, this technique is applicable for small amount of deflection in each section, and can be defined mathematically as [15]:

$$
\frac{d^{2} y}{d x^{2}}=y^{\prime \prime}=\frac{M}{E I}
$$

In which the bending moment at any position is denoted by $\mathrm{M}, \mathrm{E}$ is modulus of elasticity, $\mathrm{I}$ is the second moment of area and y" is the second derivative (curvature) of the deflection mode shape function.

In order to supplement the analysis for free vibration loading under damping-free condition, a static simulated inertia loading system may be expressed in the following form:

$K . y=\omega_{i}^{2} m y$

The right-hand side of the preceding equation represents the modal inertia force for specific mode shape (i) affecting abeam of rigidity $(\mathrm{K})$ with the purpose of generating a displacement vector $(\mathrm{y})$. 
The flexural moment at section position $\mathrm{x}(\mathrm{i}+1)$ of a beam using the particular curvature mode shape and the inertia loading condition is derived and given in the following Equation 3, [16, 17]:

$$
M_{i+1}=M_{i}+V_{i}(\Delta x)-\frac{1}{2} \int_{x_{i}}^{x_{i+1}} \omega_{i}^{2} \cdot \gamma \cdot A \cdot y(x) \cdot(\Delta x) \cdot d x
$$

Where $\gamma$ is the mass density, the cross-sectional area is (A), $\mathrm{V}$ and $\mathrm{M}$ represent the shear and bending moment, respectively.

\subsection{Damage quantification}

By using the calculated modal bending moment distribution utilizing Equation 3, the dynamic bending stiffness (EI) can be evaluated using Equation 1. From these computations, thriving insight on the location of spread damage followed by computation of its severity can be achieved.

Fig.5 shows the consecutivedrop in flexural dynamic stiffness with the applied load in beam A, as an example of applying the direct stiffness approach. The trend was approximatelysteady. A comprehensive insight on this case study has shown that the dropinflexural stiffness gonefurther from the load points to reach the supports. As a good sign of right approach, the theoretical dynamic stiffness (EI) of the starting point (intact) beam was in good agreement with the measured one. Whereas the theoretical stiffness was $840 \mathrm{kN} . \mathrm{m} 2$, the measured stiffness is $870 \mathrm{kN} . \mathrm{m} 2$. Contrary to what is visually seen,in the load step corresponding to a vertical load of $6 \mathrm{kN}$, the mid span stiffnessdecreased by almost $18 \%$,where no bending cracks were developed.

At the final load, when the beam was practically falling apart, the mid span strength reduced by $47 \%$ compared withstrength of the reference case. The beam in this stage was gone throughsevere cracking conditions.Furthermore, when the cracking area was retrofitted, the beam recovered $35 \%$ of its strength compared with the final damaged step.
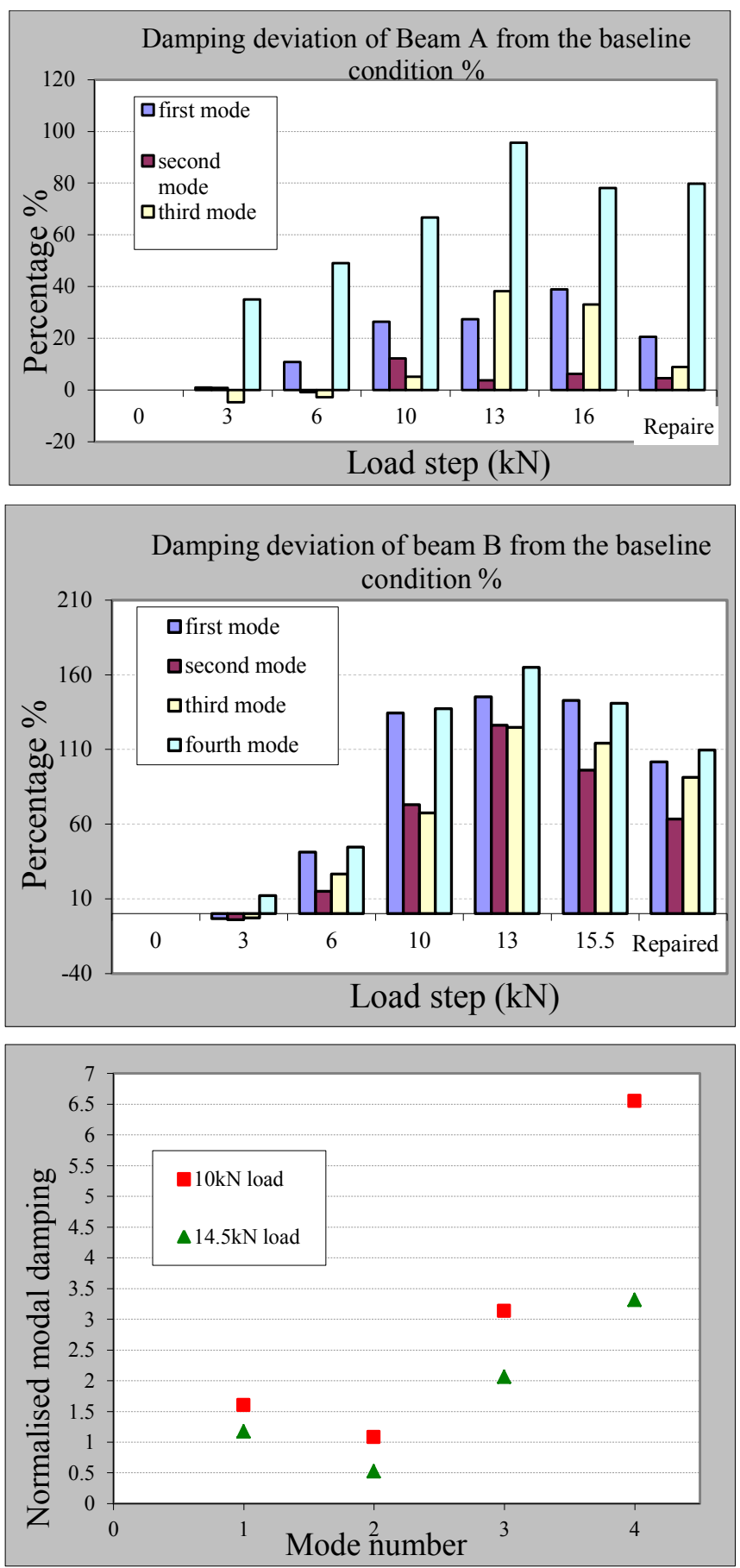

Fig. 3. Variationof modal damping for beams A, B and C for different damage conditions. 

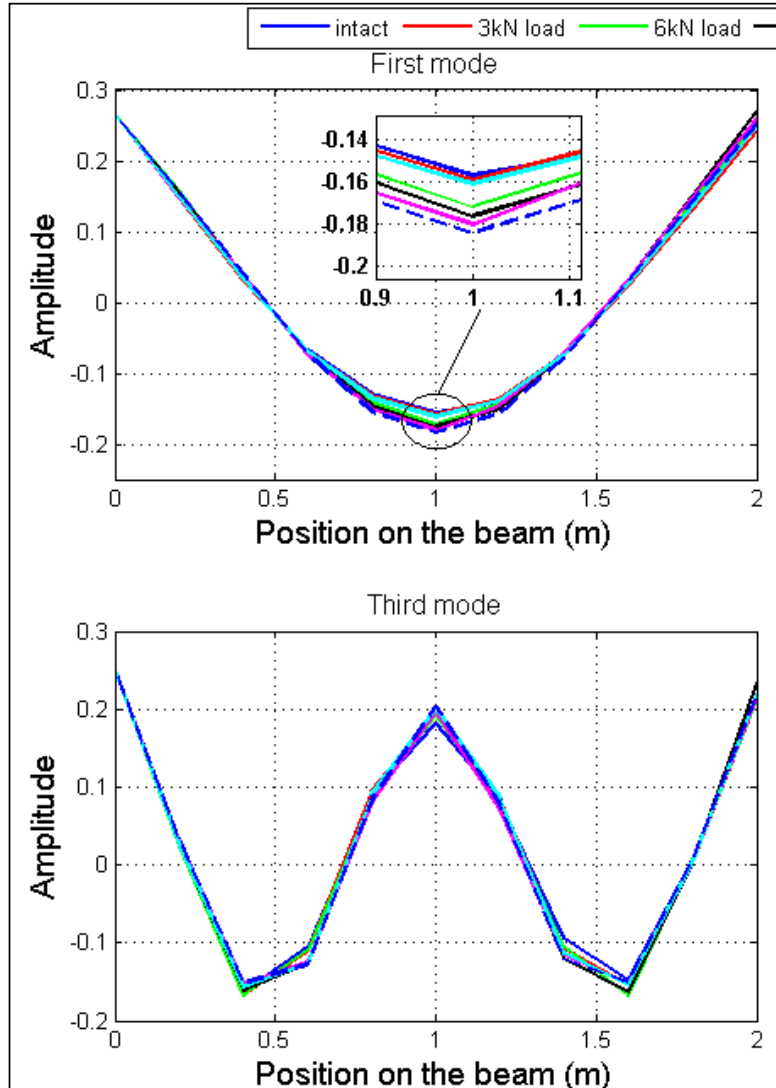

Fig. 4. Observed first four mode shapes of vibration in beam A.

\section{Summary}

SHM is now a deep-rooted area of both investigation and application along with a decent solid knowledge and research literature. There are many examples on successful applications on using vibration-based monitoring and identification systems in various disciplines. This technique can simply be applied to realworld civil engineering structure where clear and deep understanding for the structure can be gained. Of specific significance for this research is to determine the location and extent for structural degradation through combining frequencies and mode shapes of a system in a proper analytical model. A comprehensive modal parameter survey has been drawn for three RC beams deliberately damaged by active static loading. The damping parameters are usually estimated through fitting the nonlinear dynamic behaviour to a linear model of FRF. Therefore, the consistencyof outcomes may be defeated, as it has been seen in the damping ratios from this study.

When the complete deterioration was looming, the biggest resonance change in a $\mathrm{RC}$ beam was about $30 \%$ compared with the intact state condition. This drop was accompanied with maximum reduction in the flexural stiffness of about $52 \%$. Because the model of produced damage was mostly contributed to the first mode, the biggest decrease in strength and frequency basically occurred in this mode. Although such results give no concrete insight on degradation, they underpin the understanding on abnormal performance and point to faulty behaviour to correct and rectify error.
In the subject of estimating the quantity of damage, the direct stiffness method is one of the useful techniques can be advantageous used in beam structures. Mode shapes outperforms their rival modal parameters for damage sites localization, especially for the first two modes, given that the amplitude increased with the load in relation to the initial virgin state.

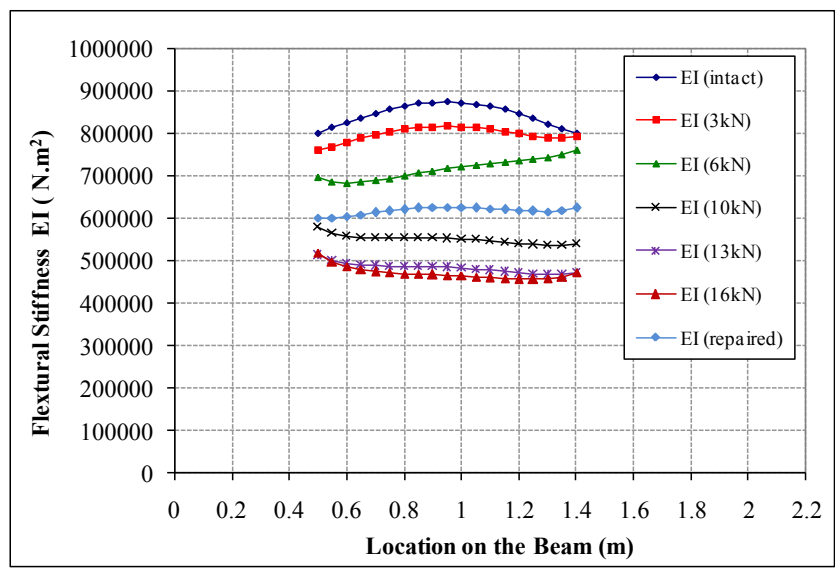

Fig. 5. Flexural stiffness distribution for beam A featured from first mode shape.

\section{References}

1. C. Farrar, D.A. Jauregui, Journal of Smart Material Structures 7, 704-719 (1998) 
2. C. Farrar, S. Doebling, D. Nix, Philosophy Transactions Royal Society 359, 131-149 (2001)

3. L. Zhengsheng, J. Swanson, A. Helmicki, V. Hunt, ASCE, Journal of Bridge Engineering 10, 2 (2005)

4. C. Farrar, K. Worden, Philosophy Transactions of Royal Society 365, 303-315 (2007)

5. E.P. Carden, P. Fanning, Journal of Structural Health Monitoring 3, 4, 355-377 (2004)

6. A.A. Al-Ghalib, PhD. Thesis, Nottingham Trent University, Nottingham, UK (2013)

7. D. Ewins, Research Studies Press Ltd., Baldock, Hertfordshire, England (2000)

8. E. Figueiredo, G. Park, J. Figueriras, C. Farrar, K. Worden, Report LA-14393, Los Alamos National Laboratory, US (2009)

9. N. Haritos, J. Owen, Journal of Structural Health Monitoring 3 2, 141-163 (2004)

10. A. Mordini , K. Savov, H. Wenzel, Structural Engineering International 17, 4, 352-358 (2007)

11. W. X. Ren, G. De Roeck, ASCE, Journal of Structural Engineering 128, 1, 96-104 (2002)

12. J. Unger, A. Teughels, G. De Roeck, The Journal of Structural Engineering 132, 11, 1691-1698 (2006)

13. H. Wenzel, Health Monitoring of Bridges, John Wiley \& Sons Ltd, West Sussex, United Kingdom (2009)

14. S. Gade, H. Herlufsen, H. Konstantin-Hansen, Application Note 3560, Brüel \& Kjær, Denmark (2005)

15. O. Huth, G. Feltrin, J. Maeck, N. Kilic, M. Motavalli, Journal of Structural Engineering, ASCE 131, 12, 1898-1910 (2005)

16. J. Maeck, G. De Roeck, Journal of Sound and Vibration 225, 1, 153-170 (1999)

17. A. Al-Ghalib, F. Mohammad, M. Rahman, J. Chilton, Damage and Repair Quantification in Reinforced Concrete Beams Using Vibration Data, Proceedings of the 4th International Conference on Concrete Solutions, September 2011, Dresden, Germany ( 2011) 